\title{
Plant Seeds Growth Prediction on Greenhouse Using Adaptive Neuro Fuzzy Inference System (ANFIS) Method
}

\author{
Siska Ayu Widiana ${ }^{*}$, Suryono Suryono ${ }^{2}$, Budi Warsito $^{3}$ \\ ${ }^{1}$ Master of Information System, Post Graduated School, Diponegoro University, Semarang, Indonesia \\ ${ }^{2}$ Department of Physics, Faculty of Science and Mathematics, Diponegoro University, Semarang, \\ Indonesia \\ ${ }^{3}$ Department of Computer Engineering, Faculty of Engineering, Diponegoro University, Semarang, \\ Indonesia
}

\begin{abstract}
Food security is a problem that every country had, especially for poor and developing countries. To improve the food security one of the solutions that can be applied is to collaborate technology and agriculture such as greenhouse. The technology that is applied to greenhouse can produce plants with good quality. Good quality plant can be predicted with prediction on the plant seeds in order to develop the plants production just as we expected. Prediction on plant seeds is using the adaptive neuro fuzzy inference system (ANFIS) model which is a combination of fuzzy and neural network. ANFIS will process the data with high complexity and it will provide the prediction result with high accuracy. Plant seeds prediction is using 65 data which divided into two data, specifically 50 training data and 15 testing data. The prediction provides accurate result and will generate $14 / 15 \times 100 \%=93.3333 \%$ precision with Mean Absolute Deviation (MAD) is 64.3391 from 15 prediction data about 4.2893, Mean Absolute Percentage Error (MAPE) is 5.3485 from 15 prediction data about 0.35657, Mean Square Deviation (MSD) is 9.159 from 15 prediction data about 0.6106 .
\end{abstract}

Keywords. Seeds Plant; Greenhouse; ANFIS; Fuzzy; Neural Network; Prediction.

\section{Introduction}

Food security is a common problem that happened in every country, especially for poor and developing country because it is related with life sustainability and citizens health for a long-term life in every country. To attain the food security, collaboration between science and technology is required [1]. Hence, the science and technology advance in agriculture industry is keep increasing. Greenhouse is an agriculture innovation which provide a

\footnotetext{
*Corresponding author: siskaayuwidiana@students.ac.id
} 
environment that can be arranged and controlled for a plant [2]. Greenhouse is intended just so the plant isn't depended with the natural environment and the environment controls can be optimalized [3]. With the touch of information systems, we can recognize plant growth characteristic very well [4]. Wireless sensor model will automatically send data to the station address. Constraints that wireless sensor system might face is the limitation on the network, especially the internet for computing and wireless control [5].

The prediction uses the ANFIS model which is a combination from neural network and fuzzy. Neural network is able to predict the growth of plant seeds in a greenhouse and obtain the accurate data near to actual [6], Meanwhile fuzzy logic is work with the rules by setting the function of membership. Based on the ANFIS model which can be developed that is ANFIS Takagi Sugeno Kang, it's advantage are simpler, works with good linear technology, and more efficient computation [7]. ANFIS is used in predicting wind velocity as the output with input variable from ANFIS model such as temperature, pressure, relative humidity, and local time. The velocity of wind is measured by time interval accurately, so the wind direction and wind velocity can be predicted [8]. ANFIS prediction was able to evaluate the impact of the temperature, humidity and viscoelastic frequency from a honey with the fusion of neural network and fuzzy model. Viscoelastics data is splitted into three, $60 \%$ is for training data, $15 \%$ is for validation data, and another $25 \%$ is for testing data [9]. ANFIS has high speed in training and is the most effective learning algorithm using a simple software. ANFIS with the function of membership will provide best accurate prediction rather than another prediction model [10].

\section{Related Work}

This research is using the ANFIS model as the method to predict the plant seeds in greenhouse. The dataset is collected and then it divided into two, for training and for ANFIS model testing. The main goal is to predict and to indicate that ANFIS model can be used for prediction purpose [11]. Advanced ANFIS model performance will be compared with statistic criteria such as coefficient determination (R2), Root Mean Square Error (RMSE) and Mean Absolute Percent Error (MAPE). The results show that the advanced ANFIS model is accurate and has the higher prediction ability [12].

Adaptive Neuro Fuzzy Inference System (ANFIS) is a combination from fuzzy logic and neural network. Fuzzy logic has an advantage in modeled the qualitative aspects of human knowledge and decision-making process by implementing a rule basis. Neural network has an advantage in recognizing a pattern, learning and practicing to solve a problem without requiring a mathematical model [13]. The fuzzy inference systems use the Takagi Sugeno Kang's (JSK) fuzzy inference system order one by considering simplicity and computation easiness. The two rules fuzzy if-then basis is shown below:

$$
\begin{aligned}
& \text { Rule } 1 \text { if }\left(x \text { is } A_{1}\right) \text { and if }\left(y \text { is } B_{1}\right) \text { then } f_{1}=\alpha_{1} x+\beta_{1} y+\gamma_{1} \\
& \text { Rule } 2 \text { if }\left(x \text { is } A_{2}\right) \text { and if }\left(y \text { is } B_{2}\right) \text { then } f_{2}=\alpha_{2} x+\beta_{2} y+\gamma_{2}
\end{aligned}
$$

Where $\mathrm{x}$ and $\mathrm{y}$ are the inputs, $\mathrm{A} 1, \mathrm{~A} 2, \mathrm{~B} 1, \mathrm{~B} 2$ are the fule of fuzzy that is already predefined, while 1 dan 2 are the outputs, $\alpha 1, \alpha 2, \beta 1, \beta 2, \gamma 1, \gamma 2$ are the linear parameters that is predefined when the training process. The ANFIS method structure has 5 layers such as fuzzyfication, rule layer, normalization layer, defuzzification layer, and sole neuro output [14]. The structure of ANFIS is shown as in Figure 1 below: 


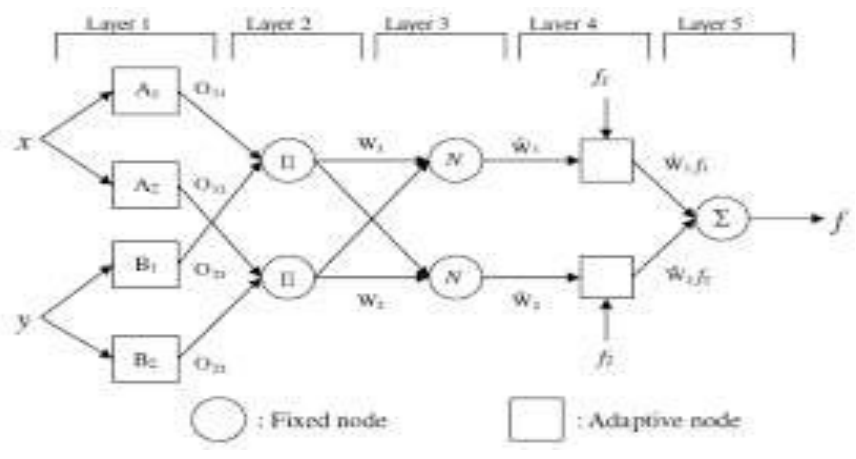

Fig. 1. Structure of ANFIS

As for the description of the ANFIS layer is as follows [15]:

a. Layer 1 (fuzzification)

Everything in this layer is a adaptive node (the parameters is able to be change) with the node functions are:

$$
\begin{aligned}
& \mathrm{O}_{1, i}=\mu_{A i}(x), \text { for } i=1,2 \text { or } \\
& \mathrm{O}_{1, i}=\mu_{B i-2}(y), \text { for } i=3,4
\end{aligned}
$$

Description:

$$
\begin{array}{ll}
\mathrm{x}, \mathrm{y} & : \text { add to } i \text { node } \\
A_{i}(x) \text { or } B_{i-2}(y) & : \text { linguistic labels to show how big or small associated with the } \\
\text { node. } & : \text { fuzzy memberships association degree } \mathrm{A}_{1}, \mathrm{~A}_{2} \text { or } \mathrm{B}_{1}, \mathrm{~B}_{2} \\
\mathrm{O}_{1, i}=\mu_{A i, 1} &
\end{array}
$$

Membership function for A can be parameterized, for example the general bell function

$$
\mu_{A}(x)=\frac{1}{1+\left|\frac{x-c}{a}\right| 2 b}
$$

With $\{a, b, c\}$ as the association of the parameter. The parameter in this layer is known as premise parameter.

b. Layer 2 (rules)

All of the node in this layer is non-adaptive (constant parameter). The output is a multiplication of all inputs that entered this layer.

The node function is:

$\mathrm{O}_{2, i}=W_{i}=\mu_{A i}(x) \cdot \mu_{B i}(y) \quad i=1,2, \ldots n$

Each node output states the fring strength of each fuzzy rules. This function can be expanded if the premise section has more than two fuzzy association. The amount of node in this layer show how much rules formed.

c. Layer 3 (Normalization)

Each of the node in this layer are a non-adaptive node that show the function of normalized firing strength, such as node - I output ratio from previous layer towards all of the outputs from the previous layer. The node function is:

$\mathrm{O}_{3, i}=\overline{W_{I}}=\frac{w i}{w 1+w 2} \quad i=1,2, . . n$

If it forms more than two rules, then the function can be expanded by dividing $\mathrm{W}_{\mathrm{t}}$ by the the total number of $\mathrm{W}$ for every rules. 
d. Layer 4 (Defuzzification)

Each of the node in this layer is an adaptive node. The node function is:

$\mathrm{O}_{4, i}=\overline{W_{I}} f_{i}=\overline{W_{I}}\left(P_{i} x x+q_{i} Y+r_{i}\right)$

Description:

$\mathrm{W}_{i} \quad$ : normalized firing strength from layer 3

$\left\{\mathrm{p}_{i} \mathrm{q}_{i} \mathrm{r}_{i}\right\} \quad$ : Association of consequent adaptive parameters.

e. Layer 5 (Neural output)

In this layers there is only one constant node, its function is to sum up all the inputs. The node function is:

$\mathrm{O}_{5, i}=\sum_{i} \overline{W_{I}} f_{i}=\frac{\sum_{i} w_{i} f_{i}}{\sum_{i} w_{i}}$

Description:

$f \quad$ : output.

\section{System Realization}

Greenhouse facility is a plant research instrument that have an advantage such as the easiness to achieve optimal plant growth and more homogeneous environment when compared to field test. With the temperature, relative humidity, carbon that is not similar with the concentration of dioxide, and irradiation that caused by microclimate. The greenhouse has been proven to introduce the impact of the disruption on growth process, production, and the plant quality. Mainly, the temperature and radiation can be considered as two main factors when related to the climate. So, in order to succeed the plant relocation, it should be noted that a plant could spent the same time in each microclimate, more likely during the comparable growth [15].

This research is using the fuzzy logic to produce more dynamic plant watering duration where the temperature and the humidity are suitable with the desired plant. When compared to conventional system, this systems is more able to process input of real values on a fuzzy scale and process it with rule basis to make decision for fuzzy output very quick and accurate [16].

Training data and testing data is processed by plant seeds prediction system using the ANFIS. ANFIS system will make the prediction with Graphical User Interface tools (GUI), GUI is able to display the prediction from reading the brightness intensity, temperature, air humidity, soil moisture, quantity of the leaves, the plant width and length of the plant stems. These data will be used as training data and it shown in table 1 .

Table 1. Data Training

\begin{tabular}{ccccccc}
\hline $\mathbf{I c}$ & $\mathbf{S}$ & $\mathbf{K u}$ & $\mathbf{K t}$ & $\mathbf{J d}$ & $\mathbf{L t}$ & $\mathbf{P b}$ \\
\hline $\mathbf{1 8 6}$ & 31.2600 & 3.3528 & 62.7188 & 2 & 5 & 2 \\
$\mathbf{2 7 6}$ & 31.3300 & 7.2464 & 63.1500 & 2 & 6 & 2.2000 \\
$\mathbf{3 7 3}$ & 31.4300 & 8.5104 & 63.7125 & 2 & 7 & 2.3000 \\
$\mathbf{4 6 2}$ & 31.5500 & 11.6410 & 64.4250 & 1 & 8 & 2.4000 \\
$\mathbf{5 9 6}$ & 30.3300 & 5.9919 & 65.4187 & 1 & 4 & 2.6000 \\
$\mathbf{6 8 6}$ & 30.5400 & 15.0645 & 66.7125 & 1 & 4 & 3 \\
$\mathbf{9 2 0}$ & 30.7800 & 24.1731 & 65.5500 & 2 & 5 & 3 \\
$\mathbf{1 1 9 6}$ & 30.9200 & 26.8292 & 65.9063 & 2 & 6 & 3.3000 \\
$\mathbf{1 2 3 9}$ & 31.1000 & 33.6129 & 65.9437 & 2 & 7 & 3.4000 \\
$\mathbf{9 2 7}$ & 31.3900 & 39.3875 & 66.1312 & 1 & 8 & 3
\end{tabular}




\begin{tabular}{ccccccc}
$\mathbf{8 9 4}$ & 31.9000 & 45.0769 & 65.1750 & 1 & 7 & 3 \\
$\mathbf{1 3 4 2}$ & 32.5000 & 43.9631 & 65.4187 & 2 & 8 & 3.5000 \\
$\mathbf{1 0 1 3}$ & 33.2100 & 51.1290 & 65.4750 & 2 & 9 & 3 \\
$\mathbf{8 8 7}$ & 34.4200 & 56.8943 & 65.0813 & 3 & 9 & 3 \\
$\mathbf{1 7 7 3}$ & 36.1200 & 56.7503 & 65.3625 & 4 & 10 & 4 \\
$\mathbf{2 7 6 4}$ & 38.4200 & 53.5553 & 64.8938 & 4 & 10 & 5 \\
$\ldots \ldots .$. & $\ldots \ldots \ldots$ & $\ldots \ldots$ & $\ldots \ldots$ &. & $\ldots$ & $\ldots \ldots$ \\
$\mathbf{3 9 4 4}$ & 33.7200 & 28.4992 & 65.6813 & 4 & 10 & 6 \\
$\mathbf{5 8 1 7}$ & 34.1400 & 38.9717 & 65.3812 & 5 & 11 & 8 \\
$\mathbf{6 0 8 7}$ & 34.1700 & 29.7721 & 65.9437 & 5 & 11 & 8.2000 \\
\hline
\end{tabular}

Total training data that is used for learning needs is 50 data and then the data will be processed with the prediction system with adaptive neuro fuzzy inference system (ANFIS) algorithm. At the system with 6 input and 1 output after the training data, load the data and choose the grid partition to generate fis with epoch is 9 and tolerance of error is 0.00005 and it will generate 7 epoch for 0.00041481 that is already qualified and the data will be tested to generate average testing error value for about 0.00041316 . The training data that is already added into the system will be proceed by predicting system ANFIS and can be shown on figure 2 .

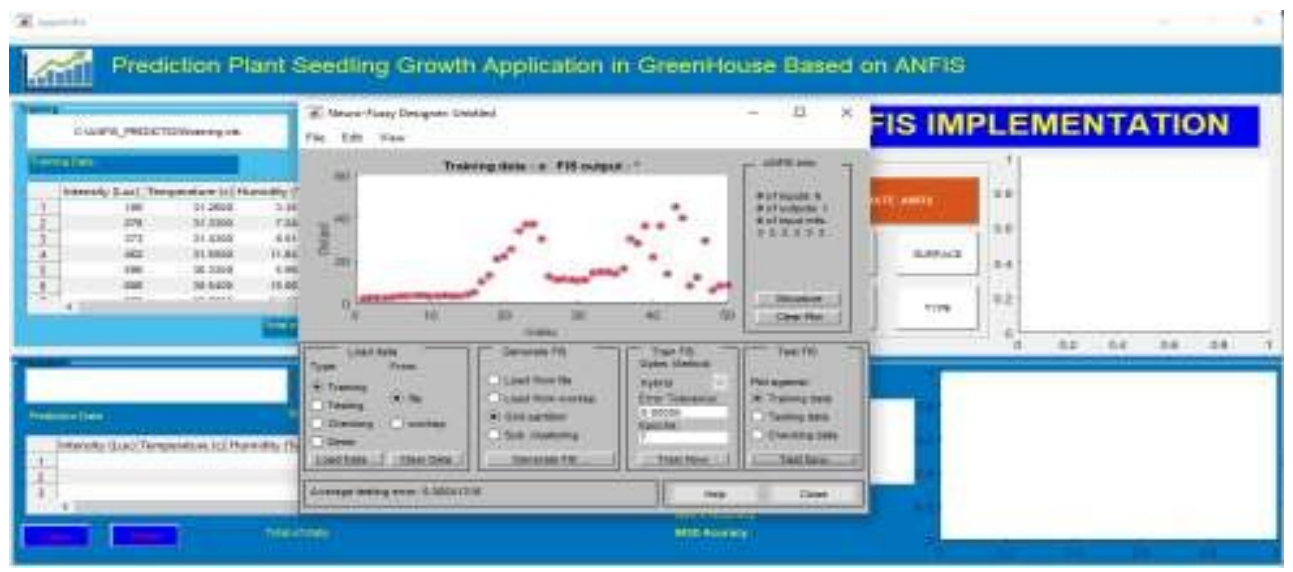

Fig. 2. Application of ANFIS Setting Neuro Fuzzy

The testing data that will be validated to be predicted is about 15 data with threshold accuracy is 16.5 . The testing data that will be validated can be shown in table 2

Table 2. Data Validation

\begin{tabular}{ccccccc}
\hline $\mathbf{I c}$ & $\mathbf{S}$ & $\mathbf{K u}$ & $\mathbf{K t}$ & $\mathbf{J d}$ & $\mathbf{L t}$ & $\mathbf{P b}$ \\
\hline $\mathbf{3 1 0 4 9}$ & 33.9400 & 40.0640 & 65.4000 & 5 & 11 & 33 \\
$\mathbf{3 8 6 8}$ & 34.2200 & 41.3748 & 65.6625 & 6 & 12 & 6 \\
$\mathbf{2 5 3 6}$ & 35.3400 & 47.6033 & 65.6625 & 7 & 12 & 4.7000 \\
$\mathbf{4 0 7 7}$ & 38.4800 & 52.8026 & 65.9812 & 7 & 13 & 6 \\
$\mathbf{4 3 6 5}$ & 38.6800 & 53.2587 & 64.5750 & 7 & 13 & 6.5000 \\
$\mathbf{1 5 2 3 4}$ & 41.0600 & 49.4112 & 63.7875 & 8 & 13 & 17 \\
$\mathbf{5 8 9 4}$ & 39.8600 & 52.5495 & 65.6250 & 8 & 13 & 8 \\
$\mathbf{5 0 8 4}$ & 38.9500 & 52.4640 & 65.7750 & 9 & 14 & 7 \\
$\mathbf{2 0 9 1 3}$ & 37.2100 & 50.3815 & 64.4437 & 10 & 15 & 23 \\
$\mathbf{5 5 2 2}$ & 39.9100 & 52.1218 & 64.6312 & 10 & 15 & 7.6000 \\
$\mathbf{9 6 0 1}$ & 37.4700 & 49.4762 & 65.4000 & 11 & 16 & 11.7000
\end{tabular}




\begin{tabular}{ccccccc}
$\mathbf{1 4 0 8 4}$ & 39.2200 & 51.9339 & 66.5437 & 12 & 17 & 16 \\
$\mathbf{5 2 8 3 8}$ & 43.5000 & 47.1392 & 64.3688 & 13 & 18 & 54.8000 \\
$\mathbf{5 5 1 5}$ & 42.3800 & 48.4853 & 65.7000 & 14 & 19 & 8 \\
$\mathbf{4 6 2 4}$ & 33.1600 & 2.0302 & 67.5000 & 15 & 20 & 6 \\
\hline
\end{tabular}

\section{Result and Discussion}

At ANFIS system, brightness intensity, temperature, air humidity, soil moisture, quantity of the leaves, and the plant width data is used as an input to predict the length of the plant stems for the output. ANFIS application is able to do the learning and prediction on greenhouse's plant seeds growth.

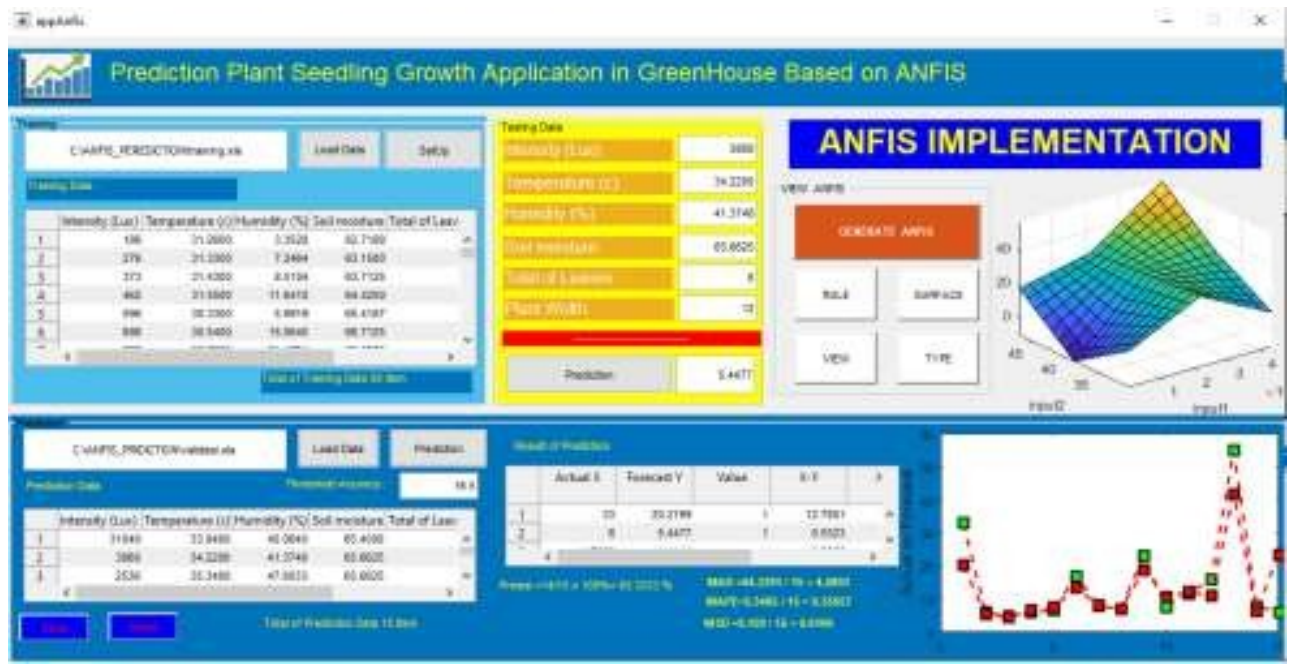

Fig. 3. Application of ANFIS

Accurate precision with the 0 number which means it's not accurate and 1 number which means accurate, from 15 data that already predicted there is only 1 data that is not accurate. The data as the result of the prediction which generate the precision $14 / 15 \times 100 \%$ $=93.3333 \%$ with Mean Absolute Deviation (MAD) is 64.3391 from 15 prediction data about 4.2893, Mean Absolute Percentage Error (MAPE) is 5.3485 from 15 prediction data about 0.35657, Mean Square Deviation (MSD) is 9.159 from 15 prediction data about 0.6106 . The prediction of the data is indicated by red colored line chart as the prediction result data, and the green colored line chart as the actual data. The prediction result data can be shown in table 3 .

Table 3. Result of Prediction

\begin{tabular}{ccccccc}
\hline $\begin{array}{c}\text { Actual } \\
(\mathbf{X})\end{array}$ & $\begin{array}{c}\text { Forecast } \\
(\mathbf{Y})\end{array}$ & $\begin{array}{c}\text { Valu } \\
\mathbf{e}\end{array}$ & $\mathbf{X}-\mathbf{Y}$ & $\mathbf{X}-\mathbf{Y}$ & $\mathbf{X}-\mathbf{Y} / \mathbf{X}$ & $(\mathbf{X}-\mathbf{Y})^{\wedge} \mathbf{2}$ \\
\hline $\mathbf{3 3}$ & 20.2390 & 1 & 12.7610 & 12.7610 & 0.3867 & 0.1495 \\
$\mathbf{6}$ & 5.4216 & 1 & 0.5784 & 0.5784 & 0.0964 & 0.0093 \\
$\mathbf{4 . 7 0 0 0}$ & 4.3868 & 1 & 0.3132 & 0.3132 & 0.0666 & 0.0044 \\
$\mathbf{6}$ & 6.4798 & 1 & -0.4798 & 0.4798 & 0.0800 & 0.0064 \\
$\mathbf{6 . 5 0 0 0}$ & 7.4802 & 1 & -0.9802 & 0.9802 & 0.1508 & 0.0227 \\
$\mathbf{1 7}$ & 13.4137 & 1 & 3.5863 & 3.5863 & 0.2110 & 0.0445 \\
$\mathbf{8}$ & 8.2024 & 1 & -0.2024 & 0.2024 & 0.0253 & $6.3995 \mathrm{e}-$
\end{tabular}




\begin{tabular}{ccccccc}
$\mathbf{7}$ & 6.9839 & 1 & 0.0161 & 0.0161 & 0.0023 & $\begin{array}{c}04 \\
5023 \mathrm{e}- \\
06\end{array}$ \\
$\mathbf{2 3}$ & 18.8363 & 1 & 4.1637 & 4.1637 & 0.1810 & 0.0328 \\
$\mathbf{7 . 6 0 0 0}$ & 10.7046 & 1 & -3.1046 & 3.1046 & 0.4085 & 0.1669 \\
$\mathbf{1 1 . 7 0 0 0}$ & 12.2441 & 1 & -0.5441 & 0.5441 & 0.0465 & 0.0022 \\
$\mathbf{1 6}$ & 10.7156 & 1 & 5.2844 & 5.2844 & 0.3303 & 0.1091 \\
$\mathbf{5 4 . 8 0 0 0}$ & 41.7261 & 1 & 13.0739 & 13.0739 & 0.2386 & 0.0569 \\
$\mathbf{8}$ & 6.1386 & 1 & 1.8614 & 1.8614 & 0.2327 & 0.0541 \\
$\mathbf{6}$ & 23.5000 & 0 & -17.5000 & 17.5000 & 2.9167 & 8.5069 \\
\hline
\end{tabular}

\section{Conclusions}

The prediction of the plant seeds growth is using the ANFIS method with some input such as, temperature, air humidity, soil moisture, quantity of the leaves, the plant width and length of the plant stems. With the training data for 50 data and testing data for 15 data, the data is proceed with fuzzification layer, rules layer, normalization layer, defuzzification layer, and neural output layer. Process to renew the parameter and determine the membership function until it generate a prediction with $14 / 15 \times 100 \%=93.3333 \%$ precision with Mean Absolute Deviation (MAD) is 64.3391 from 15 prediction data about 4.2893, Mean Absolute Percentage Error (MAPE) is 5.3485 from 15 prediction data about 0.35657, Mean Square Deviation (MSD) is 9.159 from 15 prediction data about 0.6106 .

\section{Acknowledgement}

The research and preparation of this paper was successful thanks to the exceptional support and assistance from various parties. The author would like to from the Ministry of Research, Technology and Higher Education, Republic of Indonesia, for providing this research funding assistance. Suryono and Budi Warsito, Lecturers of Master of Information System, Post Graduated School, Diponegoro University, who have guided and examined my research transcript. And all those who have supported and assisted my research.

\section{References}

1. W. Nkomoki, M. Bavorová, and J. Banout, "Adoption of sustainable agricultural practices and food security threats: Effects of land tenure in Zambia," Land use policy, vol. 78, no. March, pp. 532-538, (2018).

2. M. Ghoulem, K. El Moueddeb, E. Nehdi, R. Boukhanouf, and J. Kaiser Calautit, "Greenhouse design and cooling technologies for sustainable food cultivation in hot climates: Review of current practice and future status," Biosyst. Eng., vol. 183, pp.121-150, (2019).

3. K. Rangan and T. Vigneswaran, "An embedded systems approach to monitor green house," Proc. Int. Conf. "Recent Adv. Sp. Technol. Serv. Clim. Chang. - 2010", RSTS CC-2010, pp. 61-65, (2010).

4. R. A. Li, X. Sha, and K. Lin, "Smart greenhouse: A real-time mobile intelligent monitoring system based on WSN," IWCMC 2014 - 10th Int. Wirel. Commun. Mob. Comput. Conf., pp. 1152-1156, (2014).

5. S. Suryono, S. Sunarno, and R. Saputra, "A Fog Networks for Measuring the Physical Parameter of Greenhouse Plant," E3S Web Conf., vol. 73, pp. 1-5, (2018).

6. S. Pohan, B. Warsito, and S. Suryono, "Backpropagation artificial neural network for 
prediction plant seedling growth Backpropagation artificial neural network for prediction plant seedling growth," J. Phys. Conf. Ser., vol. 1524, pp. 1-7, (2020).

7. B. Khoshnevisan, S. Rafiee, M. Omid, and H. Mousazadeh, "Prediction of potato yield based on energy inputs using multi-layer adaptive neuro-fuzzy inference system," Meas. J. Int. Meas. Confed., vol. 47, no. 1, pp. 521-530, (2014).

8. A. Khosravi, R. N. N. Koury, L. Machado, and J. J. G. Pabon, "Prediction of wind speed and wind direction using artificial neural network, support vector regression and adaptive neuro-fuzzy inference system," Sustain. Energy Technol. Assessments, vol. 25, no. September 2017, pp. 146-160, (2018).

9. M. Oroian, "Influence of temperature, frequency and moisture content on honey viscoelastic parameters - Neural networks and adaptive neuro-fuzzy inference system prediction," LWT - Food Sci. Technol., vol. 63, no. 2, pp. 1309-1316, (2015).

10. Z. Jiang et al., "Prediction of relationship between surface area, temperature, storage time and ascorbic acid retention of fresh-cut pineapple using adaptive neuro-fuzzy inference system (ANFIS)," Postharvest Biol. Technol., vol. 113, pp. 1-7, (2016).

11. S. Jovic, J. Smigic Miladinovic, R. Micic, S. Markovic, and G. Rakic, "Analysing of exchange rate and gross domestic product (GDP) by adaptive neuro fuzzy inference system (ANFIS)," Phys. A Stat. Mech. its Appl., vol. 513, pp. 333-338, (2019).

12. M. Mostafaei, "Prediction of biodiesel fuel properties from its fatty acids composition using ANFIS approach," Fuel, vol. 229, no. February, pp. 227-234, (2018).

13. S. M. Shafaei, M. Loghavi, and S. Kamgar, "Appraisal of Takagi-Sugeno-Kang type of adaptive neuro-fuzzy inference system for draft force prediction of chisel plow implement," Comput. Electron. Agric., vol. 142, no. September, pp. 406-415, (2017).

14. R. Ata and Y. Kocyigit, "An adaptive neuro-fuzzy inference system approach for prediction of tip speed ratio in wind turbines," Expert Syst. Appl., vol. 37, no. 7, pp. 5454-5460, (2010).

15. D. Ma, N. Carpenter, H. Maki, T. U. Rehman, M. R. Tuinstra, and J. Jin, "Greenhouse environment modeling and simulation for microclimate control," Comput. Electron. Agric., vol. 162, no. November 2018, pp. 134-142, (2019).

16. R. Syam, W. H. Piarah, and B. Jaelani, "Controlling Smart Green House Using Fuzzy Logic Method,” Int. J. Smart Mater. Mechatronics, vol. 2, no. 2, pp. 116-120, (2016). 